\title{
Micromycetes-resistant colored cotton is promising material to reduce mycotoxins amounts in textiles
}

\author{
Kseniia Illarionova $^{1}$, and Sergey Grigoryev ${ }^{2, *}$ \\ ${ }^{1}$ Peter the Great St. Petersburg Polytechnic University, Saint Petersburg, Russian Federation \\ ${ }^{2}$ N. I. Vavilov All-Russian Institute of Plant Genetic Resources, Saint Petersburg, Russian Federation
}

\begin{abstract}
The aim of research was to characterize epiphyte micromycetes observed on variable cotton fibers accessions, to estimate the range of fiber destruction and select cotton, which were the most resistant to fungus damage. The accessions of differently colored Upland Cotton varieties (Gossypium hirsutum L.) evaluated: eleven cotton of natural green, twelve - of brown and eleven of conventional white color. Cotton plants have been grown in Sothern Federal District, RF. The fiber samples for the study were placed into a thermostat in sterile Petri dishes on moistened filter paper in order to stimulate the development of mycelium or sporulation of fungi naturally occurred on fibers. Incubation carried out in a thermostat at a $+24-28{ }^{\circ} \mathrm{C}$, humidity of $90-100 \%$ and exposed for 28 days. The samples examined with a microscope or binocular magnifier. Aspergillus ustus (Bainier), A. fumigatus Fresen., A. niger v. Tiegh., A. flavus Link, Penicillium aurantiogriseum Dierckx, $P$. notatum Westling, Rhizopus nigricans Ehrenb. and Alternaria alternata (Fuier) Keissler were detected. Compared with exposed white, accession of green and brown colors were significantly resistant to fungus. The mean of destruction $(\mathrm{K})$ of white cotton varied up to 0.95 , but colored accessions not exceeded 0.3 (initial destruction of the surface, not affecting internal fiber's structure).
\end{abstract}

\section{Introduction}

Cotton is a very popular raw material on the market due to its high consumer qualities [1]. The total consumption of cotton fibers from the season of 2015/16 until now has been on the rise - from $95 \mathrm{mln}$. to $108 \mathrm{mln}$. bales in 2019-20. Cotton is the main worldwide source of textile fiber, but seeds as well that widely used for oil, meals and cakes process. Cottonseeds as well as hempseed are valuable sources of functional food ingredients and prebiotics [2]. Thus, cotton is the source of a large number of industrial goods, textiles of various applications, food products and feed. Reducing the content of toxic substances in cotton products is actual. Cotton fiber, as well as the hairy surface of seeds, is a cellulose containing substrate for the life of epiphytic microflora, including cellulose-destroying

\footnotetext{
* Corresponding author: ser.grig@mail.ru
} 
micromycetes, the main danger of which are mycotoxins (aflatoxins, etc.). Mycotoxins in various amounts able to migrate to variable textile products made from cotton, as well as to products processed from cotton seeds. From textiles (clothing, bedding, home decoration, curtains, etc.) mycotoxins able to migrate into the air of residential and office premises. Secondary fungal metabolites (aflatoxins) are highly toxic substancies produced by mold Aspergillus and other species [3,4]. For both commercial and residential structures, fungal concentrations detected were often higher than currently suggested guidance values. Total indoor spore counts ranged from 610 to 1040 spores $\mathrm{m}^{-3}$ [5]. As well as chemical detoxification and physical destruction of aflatoxins in foods and feed commodities are in focus to preserve preserves the edibility of the food [6]. Therefore, preventing mycotoxins in general and aflatoxins in particular from development in products entering to human is a best approach.

\section{Purpose of investigations}

The aims of research were to observe and describe micromycetes obtained from variable cotton accessions, to estimate the destruction of fibers and select cotton accessions which are the most resistant to fungus damage.

\section{Material and methods}

The accessions of fibers of variable natural colors of cotton Upland Cotton varieties (Gossypium hirsutum L.) described in [7] were used. Eleven cotton accessions of natural green, twelve - of brown and eleven of conventional white color of fiber have been grown in Sothern Federal District, RF - in the South-Eastern part of delta of Volga in 2017-2018. To isolate the species diversity of micromycetes which occurred on cotton fiber, the method of storage materials was used. The observed fiber samples placed in a thermostat in sterile Petri dishes on moistened filter paper to stimulate the development of mycelium or sporulation of fungi located on surface of fibers. Incubation was carried out in a thermostat at a temperature of $24-28^{\circ} \mathrm{C}$ and a humidity of $90-100 \%$ for 28 days. Then the samples were examined under a microscope or binocular magnifier. The discovered mycelium was sterilely transferred to the ChapekDoks nutrient medium to identify species of fungus.

All cotton fibers accessions were studied in two factors design. First was as follow: fiber accessions have been stored for 28 days in a thermostat at a temperature of $25 \pm$ $2{ }^{\circ} \mathrm{C}$ and a relative humidity of $90-100 \%$ in order to observe the fiber's destruction after keeping in favorable for fungus development conditions. This group of accession named in the research as "exposed accessions". The alternative second design was accessions of cotton did not exposed in thermostat. These accessions mentioned in the research as "source accessions". The assessment of damage of the fibers was determined by calculating the destruction index " $K$ ", which serves as a quantitative expression of the biodegradation of cotton. Index $\mathrm{K}$ varied in limit $0.0-0.3$ corresponds to the initial destruction changes in the surface of the fiber, not affecting its internal structure. However, K exceeding 0.3 denotes initial destruction the surface, but also of the internal sections of the fiber, accompanied by initial degradation in its structure. Aseptic characteristics of the fibers in our research mean the biochemical features of the fibers that are not favorable for the development of microflora. The antiseptic properties of the fiber understood as suppressing the activity of microorganisms. The results of the analysis were processed using the ANOVA analysis of the Statistica 10.0 (StatSoft Inc., USA). 


\section{Results and discussion}

Lots of cellulose-destroying epiphyte micromycetes were detected in cotton fibers:

- Aspergillus ustus (Bainier). The fungus is widely distributed in nature and able to adapt to materials of various chemical composition, the optimum temperature of their growth is about $28^{\circ} \mathrm{C}$;

- Aspergillus fumigatus Fresen. - able to adapt to various conditions, also able to develop on polymer synthetic materials. Some strains have phytopathogenic and pathogenic properties as well.

- Aspergillus niger v. Tiegh. - is exclusively active producers of various physiologically active substances, capable of growing at low temperatures (about $0^{\circ} \mathrm{C}$ ) and at $+45^{\circ} \mathrm{C}$ as well;

- Aspergillus flavus Link, producing toxins able to dwell on synthetic polymers, widely adaptable, has peroxidase activity;

- Penicillium aurantiogriseum Dierckx, found on polymeric materials in different environmental conditions;

- Penicillium notatum Westling - distributed on variable materials including polymers in different environmental conditions, able to adapt to materials of different chemical composition. Characterized as high physiologically active;

- Rhizopus nigricans Ehrenb, capable to assimilate sugars, causing brown mold, haы a high amylolytic ability;

- Alternaria alternata (Fuier) Keissler, widely distributed in the natural environment of various climatic zones, intensively destroy cellulose, pectin and synthetic materials.

Estimation of the destruction of cotton fibers by epiphyte micromycetes accessions of cotton varieties (Gossypium L.) show, that source fiber accessions of green color was the most resistant to destruction $(\mathrm{K}=0.1$ ) by cellulose-destroying epiphyte fungus (expZ group of accessions), see figure. Accessions of brown and white colors were slightly more damaged ( $\mathrm{K}$ varied between 0.15 and 0.18 respectively).

After expose for 28 days the destruction of cotton fibers raised significantly $(p=0.05)$ and varied in range $0.3-0.95$. Commonly all accessions of conventional white color have been damaged by cellulose-destroying epiphyte fungus in maximum grade 0.95 (expW accessions, see fig. 1).

All accession of green (expZ) and brown color $(\operatorname{expB})$ were significantly most resistant to destruction by fungus. The mean grades of destruction not exceeded 0.3 .

Naturally occurring toxicant contamination of various goods (foods, textile, etc) with mycotoxins is a unique challenge to human's environment safety. Primary susceptible to aflatoxin contamination include cottonseed, as well as corn, peanuts and animal-derived foods such as milk when the animal is fed aflatoxin-contaminated feed [3]. Risks associated with aflatoxin-contaminated commodities can be reduced through the use of specific processing and decontamination procedures. Factors, which influence the effectiveness of a specific process, include the chemical stability of the mycotoxins, nature of the process, type and interaction with the food/feed matrix and interaction with multiple mycotoxins. Practical decontamination procedures suggested by [3] must: 1) inactivate, destroy, or remove the toxin, 2) not produce or leave toxic residues in the food/feed, 3) retain the nutritive value of the food/feed, 4) not alter the acceptability or the technological properties of the product, and, if possible, 5) destroy fungal spores. Aflatoxins are among the principal mycotoxins that contaminate economically important food and feed crops. Aflatoxin B1 is the most potent naturally occurring carcinogen known and is an immunosuppressant. Occurrence of aflatoxins in crops has vast economic and human health impacts worldwide [3]. We suppose, that suppress producing toxic micotocsins in the textile cotton fibres and 
cottonseed surfaces by means of development of cotton cultivars produced naturally aseptic and antiseptic fibers is promising perspective. In addition to hepatocellular carcinoma, chronic aflatoxin exposure is believed to play a role in childhood growth impairment. [McMilla 1]. Preventing mycotoxins in general and aflatoxins in particular from entering the food chain is a best approach [6]. Human exposure to micotoxins may result directly from ingestion of contaminated foods. $[3,4,5]$, as well from underwear, baby clothes, curtains, wallpapers as well. Inhalation of a biococktail products produced by fungus in residential, office, workplaces carries the danger not only of headaches, but also of more serious complications in the lungs, cardiovascular system and aggravation of psychosomatic health, which is comparable to the harm from the use of contaminated food. A possible occupational risk of aflatoxin exposure via the respiratory tract has been revealed [8].

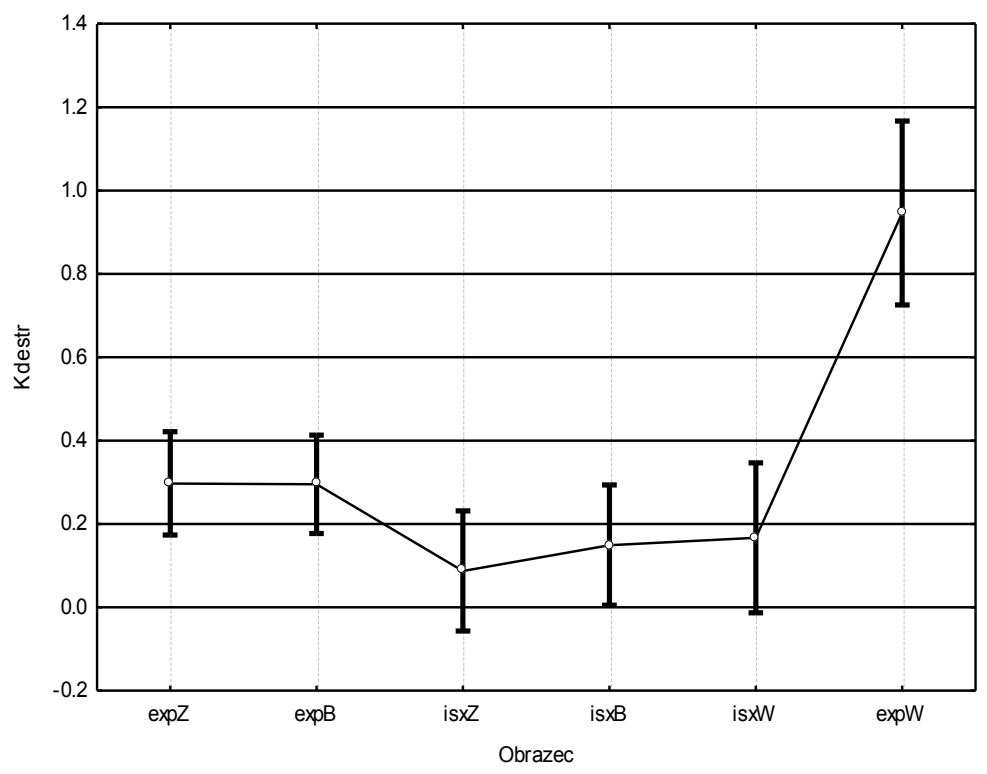

On X-axis: "expZ" denotes fiber accessions of green color exposed for 28 days, as well as "expB" - exposed of brown color, "expW" - exposed of white color, "isxB", "isxZ and "isxW" denote not exposed ("source") accessions respectively. "Kdestr" on Y-axis denotes the grades of fiber destruction. Means, vertical bars denote 0.95 confidence intervals (ANOVA). Southern Russia, 2017-2018.

Fig. 1. Naturally colored cotton fibers accessions of cotton varieties (Gossypium L.) destruction by epiphyte micromycetes.

As many as 167 microbial species were isolated from the air of examined dwellings (dwellings, offices, schools, etc.). Most frequently occurred filamentous fungi (Penicillium spp., Aspergillus spp.), and yeasts [9], as well as gram-positive cocci, endospore-forming bacilli, gram-negative bacteria. Workers at a textile plant developed interstitial lung disease. Biopsy specimens demonstrated interstitial pneumonitis and diffuse alveolar damage. Some patients got hypoxemic respiratory failure despite high-dose corticosteroid therapy. A survey of the remaining textile workers (questionnaires, chest radiographs, and pulmonary function tests) revealed supported cases. A comprehensive assessment of the work environment was performed in search of potentially toxic respirable agents [10]. It was proposed that these cases represent desquamative interstitial pneumonitis-like reactions, occupationally related to aflatoxin inhalation. In other study of textile workers [11], the effects of occupational exposure to cotton dust-with possible exposure to aflatoxin-on levels of some liver tumor biomarkers were explored. Positive reactants to 
Aspergillus niger, Aspergillus flavus, and cotton dust were significant high. However, it was observed [12] that the microbiological air pollution in spinning and weaving rooms of flax industry was significantly greater than in cotton industry. A technology of flax spinning promotes microbiological pollution in the air. A high level of mould cells in the air enhances the risk of workers' exposure to mycotoxins. Aspergillus sp. and Penicillum sp. (16.8-54\%), as well other species were found. Not only cotton and flax aterials but latex and vinyl may be contaminated with aflatoxins $[13,14]$.

As a result of our studies, naturally colored fiber cotton accessions (see table 1) have been identified, which, compared to white conventional cotton exhibits antimicrobial activity, which is probably due to its natural color (green and brown). Probably these properties determined with the chemical composition of the fiber (catechins and flavonoids) which supply cotton accessions with a color and fungi-resistant properties. Possibly, the higher antiseptic properties of beige (brown) fiber, which inhibit the activity of microorganisms and the aseptic features of green cotton, which are not favorable for the development of microflora, cause a lower degree of biodegradation of brown and green fibers compared to white cotton.

Table 1. Cotton accessions (Gossypium hirsutum L.) featured in resistance to cellulose-destroying micromycetes, aseptic characteristics of the colored fibers (not favorable for the development of fungi), Southern Russia, 2017-2018.

\begin{tabular}{|c|c|c|}
\hline Cotton accession name & Fiber color & Degree of destruction K \\
\hline Kukcha & Green & 0.209 \\
\hline Cumbazic Maron & Pink Marble (brown) & 0.104 \\
\hline Line sf & Brown & 0.279 \\
\hline Line krn & Brown & 0.300 \\
\hline LSD $_{05}{ }^{2}$ & $*$ LSD $_{05}$ - least significant difference (significance level $\left.\mathrm{p}=0.05\right)$ \\
\hline
\end{tabular}

\section{Conclusion}

Cotton is a very popular raw material on the market due to its high consumer qualities. Mycotoxins in various amounts able to migrate to variable textile products made from cotton, as well as to products processed from cotton seeds. Secondary fungal metabolites (aflatoxins) are highly toxic substancies produced by mold Aspergillus and other species. We suppose, that suppress producing toxic micotocsins in the textile cotton fibres and cottonseed surfaces by means of development of cotton cultivars produced naturally aseptic and antiseptic fibers is promising perspective. Naturally colored fiber cotton accessions have been identified, which, compared to white conventional cotton exhibits antimicrobial activity, which is probably due to its natural color (green and brown). Probably these properties determined with the chemical composition of the fiber (catechins and flavonoids) which supply cotton accessions with a color and fungi-resistant properties. Possibly, the higher antiseptic properties of beige (brown) fiber, which inhibit the activity of microorganisms and the aseptic features of green cotton, which are not favorable for the development of microflora, cause a lower degree of biodegradation of brown and green fibers compared to white cotton.

This paper publication was partially supported by the Ministry of Education and Science of the Russian Federation within the program to improve the competitiveness of Peter the Great St. Petersburg Polytechnic University (SPbPU) among the world's leading research and education centers in the 2016-2020.

The presented research performed within the framework of the State Assignment № 0662-20190001 commissioned to VIR. 


\section{References}

1. Cotton yearbook 2019. Australian cottongrower 40(6), 38-44 (2019)

2. S.V. Grigoryev, T.V. Shelenga, K.V. Illarionova, Proceedings on applied botany, genetics and breeding 180(2), 38-43 (2019). DOI: 10.30901/2227-8834-2019-2-38-43.

3. D.L. Park, Adv Exp Med Biol 504, 173-179 (2002). DOI: 10.1007/978-1-4615-0629417.

4. Y.H. Leong, A.A. Latiff, N.I. Ahmad, A. Rosma, Mycotoxin Res 28(2), 79-87 (2012). DOI: $10.1007 / \mathrm{s} 12550-012-0129-8$.

5. R.E. Gots, N.J. Layton, S.W. Pirages, AIHA J (Fairfax, Va) 64(4), 427-438 (2003). DOI: $10.1202 / 396.1$.

6. A.Y. Srour, A.M. Fakhoury, R.L. Brown, Methods Mol Biol 1542, 159-171 (2017). DOI: 10.1007/978-1-4939-6707-0_10.

7. K. Illarionova, S. Grigoryev, I. Asfondiarova, IOP Conference Series: Materials Science and Engineering, 012110 (2019). DOI:10.1088/1757-899X/497/1/012110.

8. I. Dvorackova, V. Pichova, J Toxicol Environ Health 18(1), 153-157 (1986).

9. R.L. Gorny, J. Dutkiewicz, Ann Agric Environ Med 9(1), 17-23 (2002).

10. M.D. Lougheed, J.O. Roos, W.R. Waddell, P.W. Munt, Chest 108(5), 1196-1200 (1995). DOI: 10.1378/chest.108.5.1196.

11. A. Saad-Hussein, S. Beshir, G. Moubarz, S. Elserougy, M. Ibrahim, Am J Ind Med 56(7), 818-824 (2013). DOI: 10.1002/ajim.22162.

12. J. Gościcki, L. Włodarczyk, G. Bielichowska, Med Pr 31(2), 91-97 (1980). [In Polish].

13. M. Castegnaro, H.P. Van Egmond, W.E. Paulsch, J. Michelon, J Assoc Off Anal Chem 65(6), 1520-1523 (1982).

14. E.R. Yagmurov, G.V. Kozlov, M.A. Pushkarev, Journal of Hygienic Engineering and Design 20, 60-69 (2017). 